die Lesart des Nicephorus geschlossen werden kann. Wo er mit einer in diesem Fall meistens fehlerhaften Variante allein steht, habe ich diese im allgemeinen nicht verzeichnet. Natürlich durfte ich bei den erzählenden Abschnitten, in denen Nicephorus die Fassung seiner Quellen willkürlich ändert, nicht ebenso verfahren. Hier habe ich ihn unter die Copien gestellt, und es darf niemals ex silentio geschlossen werden. Ich habe ihn nur angeführt, wenn sein Zeugnis mir einigen Nutzen zu bieten schien. In diesem Fall habe ich bei manchen Varianten von besonderem Interesse eine Verification nach der Handschrift selbst vornehmen lassen. So ist mir die oben angeführte Variante $\Sigma \alpha \dot{\eta} \nu \eta v$ von Herrn Wessely bestätigt worden.

\title{
V. Die Textgeschichte
}

Am Ziel der Untersuchung über die directe Überlieferung des Theodoret angelangt, kann ich versuchen, kurz den Ursprung und die allgemeinen Merkmale der Überarbeitung hervorzuheben, welche die meisten Handschriften erfahren hahen. Anzunehmen ist, daß die Entstellungen, die diesen Zustand des Textes herbeigefuhrt haben, im allgemeinen sehr alt sind. Theodoret schreibt mit der Emphase eines Predigers unablässig cum ira gegen die Ketzer, cum studio hinsichtlich der Rechtgläubigen; er wird nicht müde; Geschichtchen zu sammeln und zu stilisieren, in denen die priesterliche Anmaßung gegenüber der Staatsgewalt triumphiert. Durch diesen Charakterzug mußte er weit mebr als Socrates und Sozomenus, die doch noch Laien waren, die ganze Freude der Priester und Mönche ausmachen; nicht minder zog ex die Theologen durch seine reiche Urkundensammlung an. Darum ist er auch unter den Kirchenhistorikern der am meisten gelesene und abgeschriebene gewesen. Noch Photius erkennt ihm in Bezug auf die Darstellung den ersten Rang zu (Bibliotheca Cod. 31).

Frübzeitig bedeckten sich die Ränder vieler Exemplare mit Anmerkungen, und die meisten Varianten, die den minderwertigen Handschriften gemeinsam sind, reichen jedenfalls sehr weit zurück. Im allgemeinen sind die durchcorrigierten Handschriften wie L,F und wie $V$ in dem ersten Teil nieht weniger alt als die andern. In dem Bruchstück des Herrn Papadopoulos-Kerameus habe ich eine Handschrift von der Grenze des IX. und X. Jahrhunderts kennen gelernt, die bereits die meisten Entstellungen von S und G (XII. Jahrh.) enthält.

Der Zustand des Textes, der für die Archetypen n,s,v usw. vorauszusetzen ist, geht sonach auf alte Recensionen zurück. Diese rühren obne Zweifel von einer ähnlichen Bearbeitung her, wie sie auch andre 
Kirchenhistoriker betroffen hat und wie sie z. B. bei Evagrius neben der guten Überlieferung $\mathrm{A}$ eine revidierte Recension $\mathrm{z}$ hat entstehen lassen, von der die andern Handschriften abgeleitet sind; vgl. ebenfalls bei Sozomenus die beiden Recensionen BM und V (Bidez, La tradition manuscrite de Sozomène TU XXXII $2^{b}$, S. 17 ff).

Aber diese verschiedenen Recensionen, die seit dem IX. Jahrhundert und noch früher existierten, nahmen ihrerseits auch wieder Varianten altern Datums auf. Solche Varianten sind z. B. die, deren Alter durch die Übereinstimmung von Cass. oder selbst $T$ mit einer Handschriftenklasse gestützt wird (s. z. B. S. XXXVII und Lf).

Ich bringe hier einige weitere Fälle zur Sprache, wo schon die Tendenz der Überarbeitung mir den alten Ursprung zu verraten scheint.

Der merkwürdigste unter diesen Fällen findet sich vielleicht V 11, S. 300, 4 in dem Glaubensbekenntnis des Damasus. An dieser Stelle fügten die Handschriften ry, und im Anschluß an sie alle frühern Ausgaben bis auf die vorliegende, folgenden Anathematismus hinzu: $\varepsilon \ddot{\imath}$

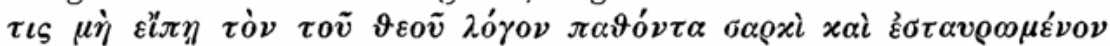

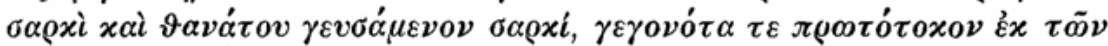

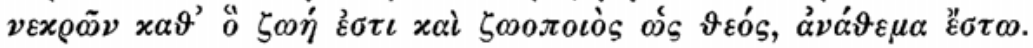

Dieser Zusatz fehlt in BVCass. $\Sigma \boldsymbol{\Lambda}$. Er fehlt auch in dem Codex F, derman hat gesehen warum - für diesen Teil zwischen den Lesarten schwankt, aber er fand sich höchst wahrscheinlich am Rande des Archetypus v, denn Nicephorus (W), dessen Handschrift ja F sehr nahe steht, hat die Einschiebung. Nur $\mathrm{A}^{\mathrm{c}}$ und $\mathrm{S}$, zwei Handschriften ohne directe Beziehungen zueinander, haben hier beide eine Scholie am Rande bewahrt, die daher aus einer sehr alten Quelle (z) in sie übergegangen ist. Diese Scholie lenkt die Aufmerksamkeit auf die Identität der Stelle mit dem

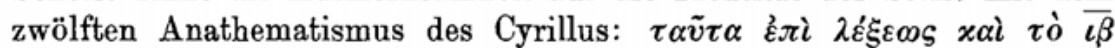

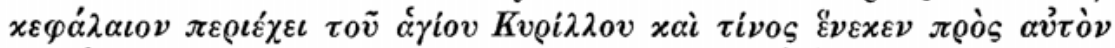

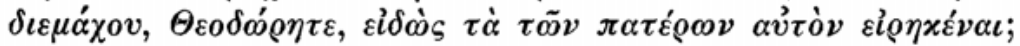

So verrät sich die Tendenz des Interpolators. Es kam ihm darauf an, unter die von Theodoret selbst anerkannte Autorität des Damasus einen der Anathematismen des Cyrillus zu stellen, gegen den der Bischof von Cyrus am meisten gekämpft hat (z. B. PG 83, 1417C 1433D). Solch ein Beweis von Unredlichkeit geht auf eine Zeit zuräck, wo der nestorianische Streit noch lebhaft war und wo die Leser noch fortfuhren, sich für oder gegen die Anathematismen des Cyrill zu erregen. Für Interpolationen äbnlicher Tendenz in Kirchenhistorikern siehe z.B. bei Schwartz, Eusebius Kirchengeschichte III, S. LXVII, sehr alte gegen Origenes gerichtete Zusätze in manchen Handschriften des Eusebius.

Das ganze Glaubensbekenntnis des Damasus bei Theodoret V 11 
ist in dieser Hinsicht durch alte Überarbeitungen mit dogmatischer Tendenz besonders interessant. Es genügt, dafür auf den kritischen Apparat zu verweisen.

Indessen ist hier noch an einige weitere Überarbeitungen zu erinnern, die einer andern Stelle entnommen sind, weil sie mir in ähnlicher Weise durch antinestorianische Besorgnisse motiviert scheinen. Sie finden sich gleichfalls in ein Glaubensbekenntnis eingeschoben 224, 1: ả $\pi 0 \delta \iota \delta o ́ v \tau \alpha$

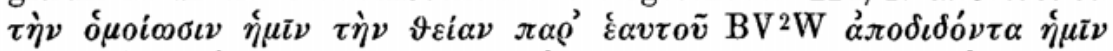

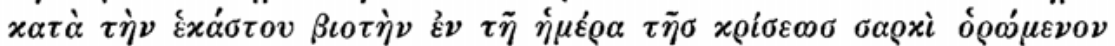

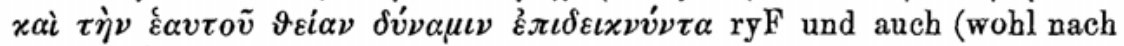
einer Randvariante) W.

Um das Motiv zur Änderung zu verstehen, sind zu vergleichen der IX.u. XI. Anathematismus des Cyrill nebst den Gegenanathematismen des Nestorius, außerdem das Glaubensbekenntnis des Theodorus von Mopsuestia (Hahn, Bibliothek der Symbole ${ }^{3}$, S. 304, 9-11). Auf der folgenden

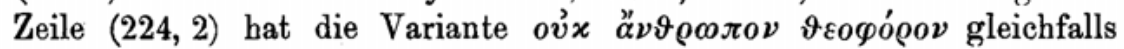
den Zweck, eine nestorianische Auslegung zu verhindern, an welche die Verfasser der Urkunde noch nicht dachten.

Natärlich haben die Änderungen nicht immer eine so specielle Tendenz, sondern sie sollen oft einfach Sinn in eine schwierige oder verderbte Stelle bringen. In dieser Hinsicht genügt es, auf den kritischen Apparat zu verweisen. Dort liegt offen zutage, was die Besserungen z. B. aus dem Symbol von Sardica II 8 gemacht haben.

Man beachte auch die kritische Bemerkung zu 255, 7, wo ich eine

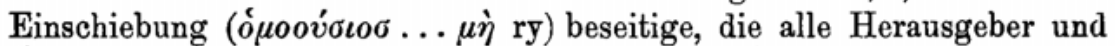
Übersetzer getäuscht hatte.

Die Textgeschichte Theodorets zeigt die im allgemeinen so häufige Tendenz, in alle Handschriften mehr und mehr die lectio facilior oder einfach die Correcturen einer neuen Recension einzuführen. Der Fall des Codex A, wo $\mathrm{A}^{\mathrm{c}}$ überall die richtigen Lesarten von $\mathrm{A}$ unterdrückt, ist dafür das charakteristischste Beispiel.

\section{Die Handschrift der Tripartita}

In der Tripartita des Theodorus Lector haben wir das Glück für viele Stellen Theodorets einen Text zu besitzen, dessen Überlieferung sich von der unsrer Handschriften seit dem sechsten Jahrhundert losgelöst hat. Nur stammt der Marcianus, die einzige Handschrift, in der die Tripartita erhalten ist, aus dem XIII. Jahrhundert, und als erste Frage drängt sich die auf, bis zu welchem Grade der von Theodorus abgeschriebene Text während jener sieben Jahrhunderte 